$\begin{array}{ll}\text { Research Square } & \text { Preprints are preliminary reports that have not undergone peer review. } \\ \text { They should not be considered conclusive, used to inform clinical practice, } \\ \text { or referenced by the media as validated information. }\end{array}$

\title{
Genome-wide investigation of histone acetyltransferase gene family and their responses to biotic and abiotic stress in foxtail millet (Setaria italica [L.] P. Beauv)
}

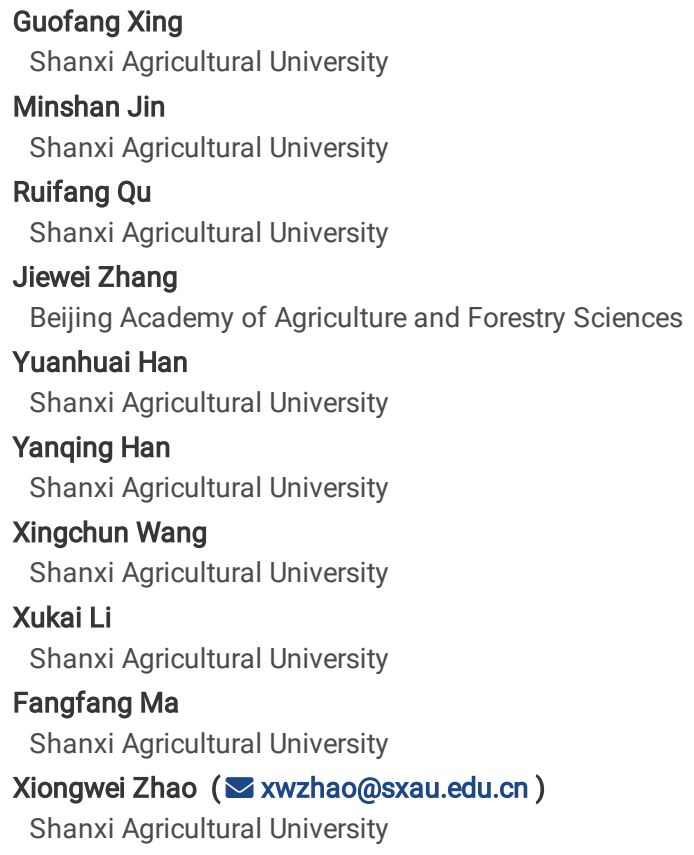




\section{Abstract}

Background: Modification of histone acetylation is a ubiquitous and reversible process in eukaryotes and prokaryotes and plays crucial roles in the regulation of gene expression in plant development and stress responses. Histone acetylation is co-regulated by histone acetyltransferase (HAT) and histone deacetylase (HDAC). HAT activity plays an essential regulatory role in a variety of growth and development processes by modifying the chromatin structure through interactions with other histone modifications and transcription factors in eukaryotic cells, affecting the transcription of genes. Comprehensive analyses of HAT genes have been performed in Arabidopsis thaliana and Oryza sativa. However, little information is available on HAT genes in foxtail millet (Setaria italica [L.] P. Beauv).

Results: In this study, 24 HAT genes (SiHATs) were identified and divided into four groups with conserved gene structures via motif compositions analysis. Phylogenetic analysis of the genes was performed to predict functional similarities between Arabidopsis thaliana, Oryza sativa, and foxtail millet; 19 and 2 orthologous gene pairs were individually identified. Moreover, all identified HAT gene pairs likely underwent purified selection based on their nonsynonymous/synonymous nucleotide substitutions. Using published transcriptome data, we found that SiHAT genes were preferentially expressed in some tissues and organs. Stress responses were also examined, and data showed that SiHAT gene transcription was influenced by drought, salt, low nitrogen, and low phosphorus stress, and that the expression of four SiHATs was altered by Sclerospora graminicola infection.

Conclusions: Results indicated that histone acetylation may play an important role in plant growth and development and stress adaptations. These findings suggest that SiHATs play specific roles in the response to abiotic stress and viral infection. This study lays a foundation for further analysis of the biological functions of SiHATs in foxtail millet.

\section{Background}

Plant nuclear DNA is organized into a DNA-protein structure called chromatin. The central nucleosome consists of 147 bp DNA, usually wrapped on an octamer of histones, which comprises two copies of the core histones $\mathrm{H} 2 \mathrm{~A}, \mathrm{H} 2 \mathrm{~B}, \mathrm{H} 3$, and $\mathrm{H} 4$ (1). Histone proteins can be extensively modified at their $\mathrm{N}$ terminal tails. The amino termini of these histones protrude from the core structure of nucleosomes and function as preferred targets for the histone modifiers involved in a series of post-translational modifications (PTMs), including acetylation, methylation, phosphorylation, ubiquitylation, and sumoylation (2, 3). These modifications of histones play very important roles in gene regulation, genome stability, and genome defense in eukaryotes, mainly by altering the structure of chromatin and/or recruiting regulatory factors $(2,4-6)$. As one of the most studied PTM mechanisms, histone acetylation alters the physical properties of nucleosomes by weakening interactions between histones and DNA (7). Histone methylation and other PTMs often create binding sites for other proteins that are bound by specific effector proteins. These can either be involved in the repression of transcription by compacting nucleosome arrays, or they can support transcription by recruiting chromatin remodeling complexes, modifying enzymes, or other complexes involved in elongation or splicing (8-11). Acetylation modification of histone proteins at the $\mathrm{N}$-terminus lysine residues plays a crucial role in regulating gene activities in eukaryotes. Acetylation of core histones is correlated with "open" chromatin configuration and is gene activated, whereas deacetylation often produces a "closed" chromatin configuration and is associated with gene repression $(7,12,13)$. Like most other PTMs, histone acetylation is reversible and is dynamically modulated by the action of histone acetyltransferases (HATs) and histone deacetylases (HDACs). Generally, HATs cause gene activation by transferring acetyl groups (CH3COO-) from acetyl-CoA onto the lysine residues of the N-terminal tails of histone proteins $(14,15)$. In contrast, HDACs regulate gene repression by removing acetyl groups from these acetylated lysine residues $(14,16)$. The targets of HATs and HDACs include H3K9, H3K14, H3K36, H4K5, H4K8, H4K12, and H4K16 (4, 16). All plant HATs are subdivided into four groups: the general control non-depressible 5-related acetyltransferase (GNAT) family; the MOZ, YBF2, SAS3, SAS2, and TIP60 (MYST) family; the camp-responsive element binding protein (CREB)-binding protein (CBP) family; and the TATA-binding protein associated factor 1 (TAF ${ }_{\|} 250$ ) family $(14,17)$. Corresponding HATs can be symbolized by the acronyms HAG, HAM, HAC, and HAF, respectively (14) and play essential roles in regulating gene expression of plant development, exogenous hormone responsive and responses to environmental stresses (18-24). The GNAT group includes three subfamilies: GCN5, elongated complex protein 3 (ELP3), and HAT1-like acetyltransferases, namely HAG1, HAG2, and HAG3 (14). The GCN5 protein is the catalytic subunit of several multi-protein HAT complexes and plays an essential role in plant development and resistance to abiotic stressors, such as heat, drought, cold, salt, and phosphate starvation $(13,17,25-26)$. In different plants, the number of HAT gene family members varies; there are 12 HATs in Arabidopsis (14), 8 in rice (27), and 32 in tomato (28). Recently, Kumar et al. (3) provided a comprehensive review of the regulation of histone acetylation during plant growth, development, and stress response.

Foxtail millet (Setaria italica [L.] P. Beauv) is one of the most important and ancient cultivated cereal crops, domesticated in northern China approximately 11,500 years ago $(29,30)$. This species exhibits specific morphological features, such as root architecture distribution, small leaf area, thick cell walls, and epidermal cell arrangement, that lend stress tolerance and high water and nutrition efficiency. These features are bolstered by a small diploid genome $(\sim 500$ $\mathrm{Mb}$ ), short life cycle, and C4 photosynthesis (31. 32). These characteristics promote foxtail millet as a model crop for exploring basic biological processes, such as plant architecture, physiology, and genome evolution (32). Additionally, the stress tolerance characteristics of foxtail millet reduce its dependence on synthetic fertilizers, pesticides, herbicides, and insecticides, highlighting it as a model crop for exploring mechanisms of stress tolerance. With the rapid development of molecular biology, the whole genome of foxtail millet has been sequenced and published by the United States Department of Energy Joint Genomic Institute and Beijing Genomics Institute of China (31,34), enabling research on the mechanisms of stress response and molecular regulation in foxtail millet and providing a foundation for genome-wide analysis of the HAT family members in this species.

In this study, we examined the whole genome of foxtail millet using bioinformatics analysis and focused on the physicochemical properties, chromosomal localization, systematic evolution, gene structure, and conserved domain of the HAT gene family.To further identify the function of the HAT genes, expression profiling upon exposure to abiotic stresses such as drought, high salinity, low nitrate, and low phosphate stress in foxtail millet was investigated, and the response of SiHATs to pathogenic Sclerospora graminicola infection was also analyzed. Overall, our results provide a foundation for further study of the 
functions of HAT genes, particularly in the responses to abiotic stresses, and will pave the way for identifying the precise role of HATs in plant growth and development.

\section{Results}

\section{Identification and chromosome mapping of the foxtail millet HAT gene family}

Extensive searches of public and proprietary transcripts and genomic databases were conducted with all previously reported HAT proteins (containing GNAT, MYST, P300/CBP, and TAF1) in rice and Arabidopsis. A total of 24 HATs were identified in foxtail millet from the Yugu1 genome after deleting redundant genes (Table 1). In addition, the position and direction of transcription of each gene were determined on foxtail millet chromosome pseudomolecules available at Phytozome (v12.1), as shown in Fig. 1. The 24 foxtail millet HAT genes were found to be distributed on nine chromosomes: eight on chromosome 2; three each on chromosomes 1, 4, and 5; two each on chromosomes 6 and 9; and one each on chromosomes 3, 7, and 8 (Table 1, Fig. 1).

Additionally, we analyzed the physical and chemical properties of all HAT family genes and encoded proteins, including the number of amino acids, molecular weight $(\mathrm{Mw})$, isoelectric point ( $\mathrm{pl})$, and subcellular location in foxtail millet. The sizes of the 24 predicted SiHAT proteins ranged from 425 aa (SiHAT9) to 5068 aa (SiHAT5), with molecular weights ranging from 38.08 (SiHAT16) to $563.55 \mathrm{kDa}$ (SiHAT5). The pl values ranged from 4.96 (SiHAT20) to 9.86 (SiHAT24). SiHAT21, SiHAT17, and SiHAT5 were determined to be neutral proteins $(-0.5<$ index of GRAVY values $<0.5)$, whereas the GRAVY values of the remaining proteins were less than 0 , indicating hydrophilic properties. Subcellular localization showed that most of the HAT genes were located in the nucleus, while two were localized to the mitochondria (SiHAT6 and SiHAT24), and two were cytoplasmic (SiHAT11 and SiHAT21). Only one protein (SiHAT5) was localized to the endoplasmic reticulum. More detailed information, including sequence, aliphatic index, instability index, and subcellular localization, are listed in Table 1. The prediction of the secondary structure of SiHAT proteins indicated that every member contained a-helix, extended chain, $\beta$-folding, and irregular curl structures. The irregular curl and a-spiral structure were the main secondary components, accounting for $30-50 \%$ of secondary structure, while $\beta$-folding accounted for only about $5 \%$ (Table 1$)$.

A chromosome region containing more than two genes within $200 \mathrm{~kb}$ is defined as tandem duplication (35). Homology analysis of SiHATs showed that there were two tandem duplication events in the foxtail millet chromosome sequences, each containing SiHAT9 and SiHAT10 on chromosome 2 and SiHAT17 and SiHAT18 on chromosome 5 (Fig. 1).

\section{Phylogenetic analysis, motif composition, and structure analysis of SiHATs}

Neighbor-joining phylogenetic analysis was performed, and the 24 SiHAT proteins were classified into Groups I, II, III, and IV, with 12, 3, 5, and 4 members, respectively (Fig. 2A). It is worth noting that most SiHAT proteins fell into sister pairs (SiHAT3 and SiHAT20, SiHAT9 and SiHAT22, SiHAT12 and SiHAT14), triplets (SiHAT8, SiHAT10, and SiHAT24) or quadruplets(SiHAT2, SiHAT15, SiHAT4, and SiHAT18) in the joint phylogenetic tree (Fig. 2A).

To obtain more insights about gene evolution, the exon-intron organization of SiHAT genes was investigated by aligning predicted coding sequences (CDS) against corresponding genomic sequences using the online service Gene Structure Display Server (GSDS). The results showed that the number of introns in the SiHAT family ranged from 2 to 23. Overall, highly similar gene structures and domains were observed for the four HAT subfamilies. In contrast, SiHAT18 and SiHAT14 did not contain both upstream and downstream regulatory regions (UTR), and SiHAT24 did not contain upstream regulatory regions. The other 21 genes exhibited upstream and downstream regulatory regions (Fig. 4, Table S3). Noticeably, the closest members from the same subgroups had a highly similar intron/exon structure regarding intron number and exon length. (Fig. 2B).

To further study the characteristic regions of SiHAT proteins, the motifs of 24 SiHAT proteins were analyzed using Multiple Expectation maximizations for Motif Elicitation (MEME). The results showed that $12 \mathrm{SiHAT}$ genes from group I belonged to the GNAT family and possessed the Bromo domain. (Fig. 2C). The

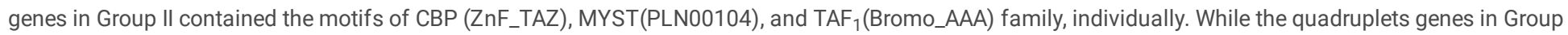
III belonged to the CBP family, contained the typical motif of HAT_KAT11 domain, PHD_SF domain, zf-TAZ and ZZ domains. The 5 genes in Group IV belonged to GNAT, CBF, and TAF families. Obviously, several HAT proteins possessed unique conserved domains, such as ELP3 in SiHAT21, HAT1 chromodomain, and Znf-C2H2 in the GNAT/MYST family. PHD (Plant Homeodomain), Znf-ZZ, and Znf-TAZ domains were observed in the CBF family. These conserved domains allowed SiHATs to interact with RNA Pol II during transcript elongation, bind with the transactivation domain of transcription factors and acetylated histone lysine residues, and perform functions by interacting with co-factors (Supplemental Table 1). Interestingly, the sister pair genes also had the same structure and conserved domain, indicating they will have the same function in foxtail millet.

\section{Phylogenetic relationship and collinearity analysis of HATs in Setaria italica, Oryza sativa, and Arabidopsis thaliana}

To better understand the phylogeny of the foxtail millet HAT gene family, the SiHATs were subjected to synteny analysis with HAT genes of the typical model plants: the dicot Arabidopsis thaliana and monocot Oryza sativa. A total of 19 SiHAT genes were synchronized with those in $O$. sativa, while two homologous pairs were identified in A. thaliana. Thus, a phylogenetic tree was constructed using the protein sequences of 24 SiHATs, 19 OsHATs, and 12 AtHATs. These 55 HAT proteins could be divided into four Clades (Fig. 3A). The results showed the phylogenetic relationship of HAT proteins between dicots and monocots. Apart from Clade II, which is the unique group of foxtail millet, the other clades included HAT proteins from the three species, suggesting that these genes existed before the divergence of monocots and dicots. Clade I was further subdivided into 4 classes, namely a, b, c, and d, with 4, 3, 5, and 4 members, respectively. Clade III further divided into classes e, f, and g (Fig. 3A).

The ratio of non-synonymous to synonymous $(\mathrm{Ka} / \mathrm{Ks}$ ) nucleotide substitutions was calculated to investigate the selective pressure on SiHATs (36). To further understand more about the phylogeny of the foxtail millet SiHAT gene family, the HAT genes of the foxtail millet underwent synteny analysis with the HAT genes of a dicotyledonous plant (Arabidopsis thaliana) and a monocotyledonous plant (Oryza sativa) (Fig. 3B). In total, 19 SiHAT genes displayed a syntenic 
relationship with those in Oryza sativa, while there were two homologous pairings in Arabidopsis thaliana. The results indicated that the genetic relationship between foxtail millet HAT genes and rice HAT genes was closer. Meanwhile, we found that some SiHAT genes were associated with rice and Arabidopsis, respectively (Fig. 3B, Supplement table 2).

\section{Cis-elements analysis of SiHAT promoters}

To further investigate the putative functions of SiHAT genes, a search in the plant promoter database (PlantCARE) was conducted in the promoter regions of 2000-bp upstream of the start codon of SiHAT genes. As shown in Fig. 4 and Supplemental Table 4, three main categories cis-elements were found in the promoter sequences of SiHAT genes. The first category was involved in phytohormones, such as abscisic acid (ABA), methyl jasmonate (MeJA), auxin, and salicylic acid (SA). The second category was associated with stresses, such as anaerobic induction, drought inducibility, low temperature responsiveness, pathogen infection, wound responsiveness, and salt inducibility. The last category was related to plant growth and development, such as zein metabolism regulation; meristem, root, endosperm inducibility (GC-motif), and abscisic acid responsive elements were found in almost all gene promoters. Importantly, all of the 24 SiHAT genes contained the light responsive element, while the MeJA-responsive element (TGACG-motif and CGTCA-motif), anoxic specific inducibility element (GC-motif) and the abscisic acid responsive element (ABRE) were found in almost gene promoters. Interestingly, instinct differences in ciselements between the sister pair genes, including in the GA and MeJA response elements, were found in the promoter of SiHAT3, whereas ABA and defense and stress response elements were found in SiHAT20. These results showed that SiHATs may have affected hormone signal responsiveness, stress adaptation, and development. No cytokinin-responsive elements in these gene promoter regions were identified.

\section{Spatial and temporal expression of SiHAT genes}

To obtain insight into the expression patterns of SiHAT genes in various tissues, a heat map was generated using the gene expression data in the foxtail millet Exp database. The results showed complex specific and overlapping SiHAT expression in various tissues and organs. For the same gene, the expression level varied among tissues and organs; for example, SiHAT17 was highly expressed in top leaves 2-3 days after the heading stage. In contrast, low or no expression signals were detected in panicles. On the other hand, the expressions of different genes were also significantly different in the same tissues and organs. For example, in the root at the filling stage, the expression of SiHAT3, SiHAT9, SiHAT13, and SiHAT22 from the GNAT gene family was significantly higher than that of other genes. Some genes were exclusively expressed in single tissues or organs; for example, SiHAT17 was expressed in leaf top after 2-3 days, and SiHAT16 was observed in immature ears. In addition, the expression patterns of three SiHATs (SiHAT3, SiHAT13, and SiHAT22) were highly expressed in all tissues at different developmental stages, whereas two SiHAT15 and SiHAT12 exhibited almost no expression in any tested tissues (Fig. 5). These results demonstrated that the expression patterns of SiHATs differed among tissues and were associated with plant growth and development.

\section{Expression analysis of SiHATs under stress}

To confirm whether the expression of SiHAT genes could be regulated by abiotic and biotic stress, we tested the effects of nitrate deficiency and phosphate deficiency, salt-alkali, and drought as abiotic stressors.

Under low nitrate conditions, the expressions of most SiHAT genes were slightly upregulated or downregulated. Most genes were downregulated after $2 \mathrm{~h}$, which was opposite at $24 \mathrm{~h}$. The expression of several genes (SiHAT17, SiHAT8, and SiHAT5) were obviously different between shoots and roots. SiHAT3 was continuously upregulated under low nitrate conditions on the shoot but only upregulated at $2 \mathrm{~h}$ on the root. This suggests that SiHAT3 likely performs the different control function during nitrate absorption and transport.

Most SiHAT genes were not strongly upregulated under low phosphate conditions, while five genes (SiHAT1, SiHAT6, SiHAT7, SiHAT19, and SiHAT21) were strongly upregulated in roots and weakly upregulated in shoots. In contrast, SiHAT17 was highly expressed in the shoot, and its expression pattern showed a sharp decline initially, then a gradual increase until returning to its original level at $24 \mathrm{~h}$ (Fig. 7).

Previous studies have reported that the expression of HAT genes was induced by drought (37). To gain further insight into the roles of SiHAT genes under drought stress, we analyzed the expression of 24 SiHATs in our RNA-seq data. The results showed that except for SiHAT15, SiHAT3, SiHAT21, and SiHAT18, the other SiHAT genes responded to drought stress with various expression patterns. Nine genes (SiHAT1, SiHAT5, SiHAT7, SiHAT8, SiHAT9, SiHAT10, SiHAT13, SiHAT19, and SiHAT22) were upregulated under drought conditions, while SiHAT17 and SiHAT15 were downregulated. SiHAT9 was highly expressed in drought-sensitive foxtail millet varieties. In response to circadian and drought treatments, SiHAT3, SiHAT9, SiHAT13, and SiHAT20 showed higher expression levels under dark conditions; meanwhile, the remaining 5 genes (SiHAT2, SiHAT8, SiHAT11, SiHAT23, and SiHAT24) were less expressed (Fig. 8).

Under salt and alkali stress, SiHAT6 was upregulated at low levels, while the other genes were significantly downregulated. The transcript levels of most genes were lower at the germinating stage than at the two-leaf one-heart stage. Only SiHAT6 and SiHAT19 showed high expression in the germinating stage. Gene expression also showed differences between sensitive and resistant varieties. At the T2 stage, the expression of 9 genes (SiHAT2, SiHAT3, SiHAT4, SiHAT5, SiHAT8, SiHAT10, SiHAT14, SiHAT18, and SiHAT20) was higher in the salt-resistant variety than in the sensitive variety, while the expression of three genes (SIHAT13, SiHAT17, and SiHAT19) showed the opposite trend (Fig. 9). SiHAT12, and SiHAT15 were the only two genes not or minimally expressed under all conditions (Figs. 6, 7, 8 and 9).

\section{SiHATs involved in Sclerospora graminicola infection}

Downy mildew disease caused by the oomycete Sclerospora graminicola (Sacc.) incident exceed to $20 \%-30 \%$ in China, and also prevalent in India, Japan and Russia, seriously reduce the yield and quality of foxtail millet $(37,38)$. We investigated the expression of SiHATs in our transcriptome sequencing data after $S$. graminicola infection. Four SiHAT genes were detected in response to infection, and their expression patterns were different. During the three-leaf-one-heart stage, the expressions of SIHAT16 and SiHAT24 were downregulated in the pathogen-resistant variety but upregulated in the sensitive variety, while SiHAT6 
and SiHAT17 were upregulated both in resistant and sensitive varieties. At the five-leaf-one-heart stage, SiHAT6 and SiHAT24 were upregulated after infection, and there was no difference between the sensitive and resistant varieties. At the seven-leaf-one-heart stage, all four genes were downregulated in the pathogen-resistant variety; however, SiHAT16 and SiHAT17 were upregulated in the sensitive variety. The other genes were not or minimally expressed (Fig. 10).

\section{Discussion}

\section{Characterization of the expanded HAT gene family in foxtail millet}

Multiple members of a specific gene family in a particular organism are natural products generated by the long evolutionary history of the organism (39). Several rounds of whole genome duplication have been found in the reported foxtail millet genomes (40). In this study, we identified and characterized 24 foxtail millet HAT genes using genome-wide analysis. Our results suggest that members of the foxtail millet HAT family are expanded as compared to Arabidopsis (12) (ref. 14), rice (19) (ref. 27), and wheat (31) (ref. 41). From the phylogenetic analysis, it can be seen that three sister pairs, one triplet, and one quadruplet were identified within the SiHAT family. However, none of these pairs were genetically linked to each other, as compared to their corresponding chromosomal locations. Conversely, all closely linked SiHAT loci, such as SiHAT9 and SiHAT10 on chromosome 2, and SiHAT17 and SiHAT18 on chromosome 5 , were not paired together into the same sister groups. Moreover, there were no sister pairs mapped on the same duplicated chromosomal blocks (Table 1 ), as described previously. Domains and motifs have been shown to be involved in various activities, including protein interaction, transcriptional activity, and DNA binding, and in this study, we found that homologous genes, such as sister pairs and quadruplets, contained conserved motifs. We also found that most SiHAT genes shared a similar exon/intron structure within the same phylogenetic group, although some differences were also observed (Figure 3B). Gene structure analysis can provide important information about gene function and evolution. It has been revealed that intron gain or loss is the result of selection pressures during evolution in plants, and genes tend to evolve into diverse exon-intron structures and perform distinct functions. Therefore, our results suggest that gene differentiation might have occurred in the SiHAT family to accomplish different biological functions under selection pressure during foxtail millet genome formation and evolution. Clades II was the specific group of foxtail millet; genes in this group showed tissue-specific expression properties, indicating it may be involved in important processes of growth and development in foxtail millet.

\section{Expression divergence between duplicated SiHAT genes}

The presence of duplicated SiHATs raises questions about their functional redundancy. According to evolutionary models, duplicated genes may undergo different selection processes: nonfunctionalization, where one copy loses function; hypofunctionalization, where one copy decreases in expression or function; neofunctionalization, where one copy gains a novel function; or subfunctionalization, where the two copies partition or specialize into distinct functions (4244). These evolutionary fates may be indicated by the divergence in expression patterns or protein structure. All SiHATs in this study appeared to be functional because they predicted a credible and complete open reading frame, and their corresponding CDNA/ESTs are available at NCBI. Evidence for divergence between the duplicate genes can be inferred from the expression pattern for the HAT quadruplet gene set. SiHAT2 was highly expressed in leaves and stems; however, the transcript levels of SiHAT4, SIHAT15, and SiHAT18 were very low. In addition, possible subfunctionalization trends were clear in the expression pattern shifts of gene pairs. For example, the mRNA abundance of SiHAT3 peaked in the root at the filling stage; however, SiHAT2O was highly expressed in the neck panicle internode. Under nitrogen deficiency, phosphorus deficiency SAS stress, the expression patterns of SiHAT3 and SiHAT20 were also different, possibly due to the large differences between the cis-elements in their promoter.

\section{Regulation of SiHAT gene expression}

Accumulating evidence has shown that HAT family genes play pivotal roles in regulating plant growth and development. Arabidopsis AtHAG1/GCN5 is one of the most widely studied and functionally characterized acetyltransferases and regulates cell differentiation, leaf and floral meristem patterning, and plant defense pathways (45). In foxtail millet, two SiHATs, SiHAT17 and SiHAT23, were grouped into the same cluster as AtHAG1/GCN5. Of these two, SiHAT17 had a similar expression pattern to AtHAG1, which is involved in low phosphorus stress, drought, and salt and alkali stress responses. Many identified HATs are involved in seed development; in this study, we found that SiHAT23 was highly expressed in germinated seeds and contained the endosperm expressive element (GCN4_motif) on its promoter. This indicates the potential role of HAT in seed development and dormancy. A review by Nguyen et al. (20) demonstrated that HAT can collaborate with ARFs to trigger auxin response and regulate root growth and development. It also showed that some SiHATs (SIHAT3, SiHAT9, SiHAT13, and SiHAT22) are highly expressed in roots and may play important roles in root development.

\section{Histone acetylation and abiotic stress responses}

The relative expression levels of HAT genes change significantly under various biotic and abiotic stresses (16). GCN5, which is involved in salt stress response, was first characterized in maize roots, and its expression increased under salt stress conditions (46). Similar expression was detected in Arabidopsis under salt stress conditions (47), and the GCN5 homologous genes SiHAT17 and SiHAT23 were also upregulated under salt stress in our experiment. OsHAC701 has been reported to respond to salt treatment in rice (27), and its homologue genes, SiHAT4 and SiHAT18, were also differentially expressed under salt and alkali stress in this study, especially at the seeding of one-heart-two-leaf. This suggests that it may play an important role in salt stress response in foxtail millet. In rice, four histone acetyltransferases, OsHAC703, OsHAG703, OsHAF701, and OsHAM701, are involved in drought responses by hyperacetylating lysine residues due to upregulation (48). In our results, their closest homolog genes also responded to the drought stress, suggesting that the two likely perform the same functions against drought stress in foxtail millet. 


\section{Histone acetylation in nitrogen and phosphorous stress responses}

Nitrogen and phosphorus are two major mineral nutrients required for plant growth and development. Histone modifications have been reported for nitrogen responses. AtHNI9, which encodes a component of the RNA polymerase II complex (IWS1), plays an important role in modulating the low nitrogen response in Arabidopsis (49). ZmCHB101 encodes a core subunit of the SWI/SNF-type ATP-dependent chromatin remodeling complex, which can bind to the promoter of the nitrogen transporter $Z m N R T 2.1 / 2$.2 through nitrate-responsive cis-elements and regulate nitrogen transport. Additionally, its RNAi lines display accelerated root growth and increased biomass under low nitrogen conditions compared to wild type plants (50). This suggests that histone modification plays an important role in regulating plant growth and development under low nitrogen conditions. In foxtail millet, we found that some HAT genes were responsive to low nitrogen stress, and the expression patterns were different between the different groups, indicating that the mechanism of histone regulation is complex, and further investigation is needed to clarify the function of SiHATs under low nitrate responsive.

The histone acetyltransferase AtGCN5 is required for the activation of several genes under low Pi conditions, including At4 and AtWRKY6. Mutation of AtGCN5 reduces $\mathrm{Pi}$ concentration in Arabidopsis plant and impairs $\mathrm{Pi}$ accumulation between roots and shoots. This indicates that GCN5-mediated histone acetylation regulates the phosphate starvation response through the At4-miR399-PHO2 pathway (51). We found that the GCN5 homologue gene SiHAT17 responded to low phosphorus stress and was highly expressed in roots under low phosphorus conditions. However, the expression pattern was reversed in the shoot, suggesting a difference in histone modification between shoot and root under low phosphate conditions in foxtail millet. These findings provide evidence that the mechanisms regulating the responses of shoots and roots to low phosphate stress are different, and further investigation should be conducted on the role of SiHAG5 in the regulation of low phosphorus stress.

\section{Histone acetylation and biotic stress responses}

Histone acetylation dynamics by HATs and HDACs is a key regulatory epigenetic mechanism that ultimately regulates plant development, hormone homeostasis, stress response and defense responses in plants (3, 52-55). In this study, we identified 24 HATs in foxtail millet and found more abiotic and biotic responsive elements in their promoters, and most SiHAT genes responded to nitrogen deficiency, phosphorus deficiency, drought, salt alkali stress, and infection of Sclerospora graminicola. All of these favorable conditions indicate that these proteins may be involved in plant responses to abiotic and biotic stress; however, the mechanisms behind this stress response, and how HATs interact with other histone modifiers, remain unclear. A future study should include transgenic research and protein interaction of key genes related to abiotic and biotic stress, and use of new CRISPR-Cas 9 technology may improve the process.

\section{Conclusions}

Recent studies show that histone acetylation and deacetylation play essential roles in the regulation of plant growth and development, as well as in responses to stress. Analyses of various mutations of HAT and HDAC genes in Arabidopsis have revealed the function of histone acetylation/deacetylation in plant development. Here, we screened 24 histone acetylation transferase genes that may be involved in abiotic and biotic stress response in foxtail millet. We identified their chromosomal locations, protein structures, gene duplications, promoters, and conserved motifs. Phylogenetic and synteny comparisons between AtHATs, OsHATs, and SiHATs were performed, and the potential roles of SiHATs in the development and growth of foxtail millet were investigated based on our previously published RNA-seq data. Candidate SiHATs involved in responses to low nitrate, low phosphate, drought, salt response, and $S$. graminicola infection were examined and assessed through the analysis of promoter elements. Our results lay a foundation for research regarding histone modification stress response regulation in foxtail millet and similar crops. Further studies, including biological experiments, will be required to confirm the functions of the candidate genes, identify the interacting HATs and HDACs, and clarify the molecular mechanisms by which histone acetylation/deacetylation affects various biological processes. This study served as a reference to improve the stress resistance of plants.

\section{Methods}

\section{Sequence retrieval and identification of HAT genes.}

We retrieved data containing sequence IDs, protein sequences, genomic sequences, and conserved domain sequences (CDS) of foxtail millet and rice from Phytozome (https://phytozome.jgi.doe.gov/pz/portal.html V12.1). The AtHAT gene sequence from Arabidopsis thaliana was retrieved from Uniprot (https://www.uniprot.org), and the Hidden Markov Model was used to identify foxtail millet genes in a protein database with the BLASTP program (P value = 0.001). The obtained proteins were run through the Pfam database and SMART (http://pfam.xfam.org/) to eliminate the sequences not containing complete HAT domains. Then, the conserved domains were checked by the conserved domain database (CDD) program (https://www.ncbi.nlm.nih.gov/Structure/cdd/wrpsb.cgi) in order to confirm the presence of the complete HAT domain. Then, all candidate sequences were verified using BLASTn (https://blast.ncbi.nlm.nih.gov/Blast.cgi) and HMMER (http://www.hmmer.org). Finally, the cis-acting elements were predicted for 2000 bp upstream of all extended genes using PlantCARE (http:// bioinformatics.psb.ugent.be/webtools/plantcare/html/).

\section{Phylogenetic analysis of HATs.}

The exon/intron structures of the SiHATs were deduced from alignments of cDNA and BAC genomic sequences using gene structure displayer (http://gsds.cbi.pku.edu.cn/). Multiple-sequence alignments of SiHAT proteins were carried out using the Clustal W (version 2.0) program (56). The protein sequences of histone acetyltransferase proteins in Arabidopsis and rice were obtained from the TIGR database. Then, phylogenetic analysis was performed with MEGA7.0 (57) using the neighbor-joining method (1000 bootstrap replications), and the results were visualized in Itol (58). 
Swiss-Prot, physicochemical properties, subcellular localization, pl, and Mw of the putative SiHATs were calculated using the ExPASy online tool (https://www.genscript.com/psort.htm). Secondary protein structures were predicted using SOPMA software (https://prabi.ibcp.fr/htm/site/web/home).

Chromosome information was obtained from Phytozome (http://www.phytozome.net), and a chromosomal location map of the genes was generated by TBtools (https://github. com/CJ-Chen/TBtools). A distinctive name was given to each SiHAT according to its initial position on the chromosomes. Conserved motif analysis of foxtail millet HAT protein sequences was conducted using the MEME suite 4.11 .1 software (http://meme.nbcr.net/meme/) (59), with the motif width set to 6-300 and the maximum number of motifs set to 20 . The results were visualized using TBtools.

\section{Analysis of duplication and synteny of HAT family genes in Arabidopsis, foxtail millet, and rice.}

In order to confirm the gene duplication events of HATs, we investigated the ancient duplication events between Arabidopsis thaliana, Orazy sativa, and Setaria italica. The non-synonymous substitution rate (Ka), synonymous substitution rate (Ks), and $\mathrm{Ka} / \mathrm{Ks}$ ratio for each pair of duplicated genes among $A$. thaliana, O. Sativa, and S. italica were computed between pairs of homologous genes using the $\mathrm{Ka} / \mathrm{Ks}$ calculator in TBtools with default settings. Multiple collinear scanning was used to simultaneously detect the homologous genetic relationships between different species.

\section{Promoter analysis of SiHAT genes}

The cis-acting elements of promoters are essential for determining tissue-specific expression and are involved in the regulation of gene expression under abiotic stress. A region 2000 bp upstream of the start codon (ATG) was investigated from the reference genome sequence of foxtail millet. The online software PlantCARE (http: bioinformatics.psb.ugent.be/webtools/plantcare/html) was used to search for cis-acting regulatory elements in the promoters of SiHATs.

\section{Analysis of gene expression profiles.}

Data on the expression of SiHAT genes in 23 tissue types of the foxtail millet cultivar JG21 were obtained from a published foxtail millet database (http://foxtail-millet.biocloud.net/home) (32). These tissues included leaves at the seeding and different filling stages, seeds at various maturation stages, roots at the filling stage, panicles, and neck panicles, detailed as in Supplemental table 4. Detailed information can be found in Supplemental Table 3. All RNA sequencing library preparations were conducted as described in our previous study (32). The time and spatial expression data for SiHATs were collected from our Electronic Fluorescent Pictograph (xEFP) program (http://sky.sxau.edu.cn/MDSi.html) and the aforementioned database (32). Heatmap Illustrator Hemil v.1.0, was used to plot the heat map of gene expression (60).

\section{Tissue preparation.}

Seeds from the foxtail millet cultivar Jingu21 were germinated on paper rolls for three days, then incubated at $25^{\circ} \mathrm{C}$ (day) or $22{ }^{\circ} \mathrm{C}$ (night) on a $16: 8 \mathrm{~h}$ light:dark schedule for three weeks. Seedlings at the five-leaf stage were transferred to Hoagland solution with $1 \mathrm{mM} \mathrm{KH}_{2} \mathrm{PO}_{4}(\mathrm{Control} \mathrm{CK})$ or $5 \mu \mathrm{M} \mathrm{KH_{2 }} \mathrm{PO}_{4}$ (low phosphorus treatment). The shoot and root tissues were harvested at $0.5,2,6,12,24$, and $72 \mathrm{~h}$ after treatment.

For the nitrate experiment, the plants were prepared as before. In the control treatment, (normal nitrate; NN) the nitrate concentration was $2.0 \mathrm{mM}$, and in low nitrate (LN), it was $0.2 \mathrm{mM}$. After $10 \mathrm{~min}, 30 \mathrm{~min}$, and 2, 8, 24, and $72 \mathrm{~h}$, the shoot and root tissue were harvested. Special care was taken to characterize the materials used, and three replicates were harvested for RNA extraction.

The seedlings were grown in distilled water for $14 \mathrm{~d}$, then subjected to remaining stress treatments. For the drought treatment, two different varieties of foxtail millet were used: AN04 (drought resistant) and Yugu 1 (drought sensitive). Conditions included different circadian stages as morning (8:00-9:00), noon (12:00-13:00), evening (18:00-19:00). Leaf tissues from plants under the control and drought conditions were collected in the morning, noon, and evening after three days of treatment.

For the salt and saline treatments, salt sensitive (B355) and salt tolerant (B103) seeds were germinated in Hoagland nutrient solution supplemented with 200 $\mathrm{mM} \mathrm{NaCl}$ and a $30 \mathrm{mM}$ mixture of $\mathrm{Na}_{2} \mathrm{CO}_{3}$ and $\mathrm{NaHCO}_{3}$ to represent salt and saline-alkaline stress; they were germinated in distilled water as the control treatment. Plants were harvested after $3 \mathrm{~d}$ (at the germination stage) and two weeks (two-leaf one-heart stage).

For the S. graminicola infection experiments, Jingu21 (pathogen-sensitive) and Jingu42 (pathogen-resistant) cultivars were germinated as described for the phosphate experiments. They were then infected at three growth periods: the 3-leaf, 5-leaf, and 7-leaf stages. The univision plant was set as the control, and three replicates were harvested for each genotype between 8:00 and 9:00 am.

\section{Declarations}

\section{Ethical approval and consent to participate]}

Experimental research complied with local and national regulations for using plants.

\section{Consent for publication:}

Not applicable.

\section{Availability of data and materials:}

All datasets supporting the results of this study are included within the article and its supplementary information. 
The authors declare that they have no competing interests.

\section{Funding:}

This work was supported by Shanxi Scholarship Council of China (Grant No. 2021-071); the Natural Science Foundation of Shanxi Province (Grant No. 20210302123364); National Science Foundation of China (Grant No.31901598, 32070366)

\section{Author contributions:}

MJ and RQ performed the bioinformatics analyses; GX drafted the manuscript; $\mathrm{XZ}, \mathrm{YH}$, and FM analyzed the transcriptomic data; $\mathrm{JZ}$, $\mathrm{YH}$, and $\mathrm{MJ}$ planted the crops and collected materials; $\mathrm{XZ}, \mathrm{XL}$, and $\mathrm{XW}$ supervised the research and modified the manuscript. $\mathrm{GX}$ and $\mathrm{MJ}$ contributed equally. All authors have read and approved the final manuscript.

\section{Acknowledgements:}

We would like to thank Doctor Siyu Hou and Li Zhang, University of Shanxi Agricultural University, China, for discussions and help with the manuscript.

\section{References}

1. Luger K, Mäder AW, Richmond RK, Sargent DF, Richmond TJ. Crystal structure of the nucleosome core particle at 2.8 $\AA$ resolution. Nature. 1997;389:25160 .

2. Kouzarides T. Chromatin modifications and their function. Cell. 2007;128:693-705.

3. Kumar V, Thakur JK, Prasad M. Histone acetylation dynamics regulating plant development and stress responses. Cell Molec Life Sci. 2021;78:4467-86.

4. Zhang K, Sridhar VV, Zhu J, Kapoor A, Zhu JK. Distinctive core histone post-translational modification patterns in Arabidopsis thaliana. PLoS One. 2007;11:e1210.

5. Chinnusamy V, Zhu JK. Epigenetic regulation of stress responses in plants. Curr Opin Plant Biol. 2009;12:133-39.

6. Portela A, Esteller M. Epigenetic modifications and human disease. Nature Biotechnol. 2010;28:1057-68.

7. Grunstein M. Histone acetylation in chromatin structure and transcription. Nature. 1997;389:349-52.

8. Lin G, Zhou Y, Li M, Fang Y. Histone 3 lysine 36 to methionine mutations stably interact with and sequester SDG8 in Arabidopsis thaliana. Sci China Life Sci. 2018;61:225-34.

9. Liu R, Li X, Chen W, Du J. Structure and mechanism of plant histone mark readers. Sci China Life Sci. 2018;61:170-7.

10. Qian S, Lv X, Scheid RN, Lu L, Yang Z, Chen W, et al. Dual recognition of H3K4me3 and H3K27me3 by a plant histone reader SHL. Nat Commun. 2018;9:2425.

11. Yang Z, Qian S, Scheid RN, Lu L, Chen X, Liu R, et al. EBS is a bivalent histone reader that regulates floral phase transition in Arabidopsis. Nat Genet. 2018;50:1247-53.

12. Shahbazian MD, Grunstein M. Functions of site-specific histone acetylation and deacetylation. Annu Rev Biochem. 2007;76:75-100.

13. Yuan LY, Liu XC, Luo M, Yang SG, Wu KQ. Involvement of histone modification in plant abiotic stress responses. J Int Plant Biol. 2013;55:892-901.

14. Pandey R, Müller A, Napoli CA, Selinger DA, Pikaard CS, Richards EJ, et al. Analysis of histone acetyl-transferase and histone deacetylase families of Arabidopsis thaliana suggests functional diversification of chromatin modification among multicellular eukaryotes. Nucleic Acids Res 2002;30:5036-55.

15. Aquea F, Timmermann T, Arce-Johnson P. Analysis of histone acetyltransferase and deacetylase families of Vitis vinifera. Plant Physiol Biochem. 2010;48:194-9.

16. Bjerling P, Silverstein RA, Thon G, Caudy A, Grewal S, Ekwall K. Functional divergence between histone deacetylases in fission yeast by distinct cellular localization and in vivo specificity. Mol Cell Biol. 2002;22:2170-81.

17. Perrella G, Consiglio MF, Aiese-Cigliano R, Cremona G, Sanchez-Moran E, Barra L, et al. Histone hyperacetylation affects meiotic recombination and chromosome segregation in Arabidopsis. Plant J. 2010;62:796-806.

18. Liu X, Yang S, Yu CW, Chen CY, Wu K. Histone acetylation and plant development. Enzymes. 2016;40:173-99.

19. Papaefthimiou D, Likotrafiti E, Kapazoglou A, Bladenopoulos K, Tsaftaris A. Epigenetic chromatin modifiers in barley: III. Isolation and characterization of the barley GNAT-MYST family of histone acetyltransferases and responses to exogenous ABA. Plant Physiol Biochem. 2010;48:98-107.

20. Nguyen CT, Tran G, Nguyen NH. Homeostasis of histone acetylation is critical for auxin signaling and root morphogenesis. Plant Mol Biol. 2020;103:1-7.

21. Wang Z, Cao H, Sun Y, Li X, Chen F, Carles A, et al. Arabidopsis paired amphipathic helix proteins SNL1 and SNL2 redundantly regulate primary seed dormancy via abscisic acid ethylene antagonism mediated by histone deacetylation. Plant Cell. 2013;25:149-66.

22. Kaldis A, Tsementzi D, Tanriverdi O, Vlachonasios KE. Arabidopsis thaliana transcriptional co-activators ADA2b and SGF29a are implicated in salt stress responses. Planta. 2020;233:749-62.

23. Zhou Y, Tan B, Luo M, Li Y, Liu C, Chen C, et al. HISTONE DEACETYLASE19 interacts with HSL1 and participates in the repression of seed maturation genes in Arabidopsis seedlings. Plant Cell. 2013;25:134-48.

24. Li C, Liu D, Lin Z, Guan B, Liu D, Yang L, et al. Histone acetylation modification affects cell wall degradation and aerenchyma formation in wheat seminal roots under waterlogging. Plant Growth Regul. 2019;87:149-63. 
25. Hu Z, Song N, Zheng M, Liu X, Liu Z, Xing J, et al. Histone acetyltransferase GCN5 is essential for heat stress-responsive gene activation and thermotolerance in Arabidopsis. Plant J. 2015;84:1178-91.

26. Mao YP, Pavangadkar KA, Thomashow MT, Triezenberg SJ. Physical and functional interactions of Arabidopsis ADA2 transcriptional coactivator proteins with the acetyltransferase GCN5 and with the cold-induced transcription factor CBF1. Miochimica et Biophysica Acta. 2006:1759: 69-79.

27. Liu X, Luo M, Zhang W, Zhao JH, Zhang JX, Wu KQ, et al. Histone acetyltransferases in rice (Oryza sativa L.): phylogenetic analysis, subcellular localization and expression. BMC Plant Biol. 2012;12:145.

28. Cigliano RA, Sanseverino W, Cremona G, Ercolano MR, Conicella C, Consiglio FM. Genome-wide analysis of histone modifiers in tomato: gaining an insight into their developmental roles. BMC Genom. 2013;14:57.

29. Hu H, Mauro-Herrera M, Doust AN. Domestication and improvement in the model C4 grass Setaria. Front Plant Sci. 2018;9:719.

30. Lu H, Zhang J, Liu KB, Wu N, Li Y, Zhou K, et al. Earliest domestication of common millet (Panicum miliaceum) in East Asia extended to 10,000 years ago. Proc Natl Acad Sci U S A. 2009;106: 7367-72.

31. Zhang GY, Liu X, Quan ZW, Cheng SF, Xu X, Pan SK, et al. Genome sequence of foxtail millet (Setaria italica) provides insights into grass evolution and biofuel potential. Nat Biotechnol. 2012;30:549-54.

32. Yang Z, Zhang H, Li X, Shen H, Gao J, Hou S, et al. A mini foxtail millet with an Arabidopsis-like life cycle as a C4 model system. Nat Plants. 2020;6:116778.

33. Doust AN, Kellogg EA, Devos KM, Bennetzen JL. Foxtail millet: a sequence-driven grass model system. Plant Physiol. 2009;149:137-41.

34. Bennetzen JL, Schmutz J, Wang H, Percifield R, Hawkins J, Pontaroli AC, et al. Reference genome sequence of the model plant Setaria. Nat Biotechnol. 2012;30:555-61.

35. Holub EB. The arms race is ancient history in Arabidopsis, the wildflower. Nature Rev Genet. 2001;12:516-27.

36. Anton N, Makova KD, Li WH. The Ka/Ks ratio test for assessing the protein-coding potential of genomic regions: An empirical and simulation study. Genome Res. 2002;12:198-202.

37. Obayashi M, Hiraka Y, Abe A, Yaegashi H, Natsume S, Kikuchi H, et al. Genome analysis of the foxtail millet pathogen Sclerospora graminicola reveals the complex effector repertoire of graminicolous downy mildews. BMC Genom. 2017;18:897.

38. Li RJ, Han YQ, Zhang Q, Chang GR, Han YH, Li XK, et al. Transcriptome profiling analysis reveals co-regulation of hormone pathways in foxtail millet during Sclerospora graminicola infection. Int J Mol Sci. 2020;21:1226.

39. Xing HY, Pudake RN, Guo GG, Xing GF, Hu ZR, Zhang YR, et al. Genome-wide identification and expression profile of Auxin Response Factor (ARF) gene family in maize. BMC Genom, 2011,12:178

40. Paterson AH, Bowers J, Peterson D, Estill J, Chapman B. Structure and evolution of cereal genomes. Curr Opin Genet Dev. 2003;13:644-50.

41. Gao SQ, Li LZ, Han XL, Liu TT, Jin P, Cai LN, et al. Genome-wide identification of the histone acetyltransferase gene family in Triticum aestivum. BMC Genom. 2021;22:49

42. Lynch M, Conery JS. The evolutionary fate and consequences of duplicate genes. Science. 2000;290:1151-5

43. Otto SP, Yong P. The evolution of gene duplicates. Adv Genet. 2002;46:451-83.

44. Duarte JM, Cui L, Wall PK, Zhang Q, Zhang X, Leebens-Mack J, et al. Expression pattern shifts following duplication indicative of subfunctionalization and neofunctionalization in regulatory genes of Arabidopsis. Mol Biol Evol. 2006;23:469-78.

45. Servet C, Silva NC, Zhou DX. Histone acetyltransferase AtGCN5/HAG1 is a versatile regulator of developmental and inducible gene expression in Arabidopsis. Mol Plant. 2010;3:670-7.

46. Li H, Yan S, Zhao L, Tan J, Zhang Q, Gao F, et al. Histone acetylation associated up-regulation of the cell wall related genes is involved in salt stress induced maize root swelling. BMC Plant Biol. 2014;14:1-14.

47. Zheng M, Liu XB, Lin JC, Liu XY, Wang Z, Xin M, et al. Histone acetyltransferase GCN5 contributes to cell wall integrity and salt stress tolerance by altering the expression of cellulose synthesis genes. Plant J. 2019;97:587-602.

48. Fang H, Liu X, Thorn G, Duan J, Tian L. Expression analysis of histone acetyltransferases in rice under drought stress. Biochem Biophys Res Commun. 2014;443:400-5.

49. Widiez T, El Kafafi S, Girin T, Berr A, Ruffel S, Krouk G, et al. High nitrogen insensitive 9 (HNI9)-mediated systemic repression of root $\mathrm{NO}_{3}{ }^{-}$uptake is associated with changes in histone methylation. Proc Natl Acad Sci U S A. 2011;108:13329-34.

50. Meng X, Yu X, Wu Y, Kim DH, Nan N, Cong W, et al. Chromatin remodeling protein ZmCHB101 regulates nitrate-responsive gene expression in maize. Front Plant Sci. 2020;11:52.

51. Wang T, Xing J, Liu Z, Zheng M, Yao Y, Hu Z, et al. Histone acetyltransferase GCN5-mediated regulation of long non-coding RNA At4 contributes to phosphate starvation response in Arabidopsis. J Exp Bot. 2019;70:6337-48.

52. Kim S, Piquerez SJM, Ramirez-Prado JS, Mastorakis E, Veluchamy A, Latrasse D, et al. GCN5 modulates salicylic acid homeostasis by regulating H3K14ac levels at the $5^{\prime}$ and $3^{\prime}$ ends of its target genes. Nucleic Acids Res. 2020;48:5953-66.

53. Wang T, Xing J, Liu X, Yao Y, Hu Z, Peng H, et al. GCN5 contributes to stem cuticular wax biosynthesis by histone acetylation of CER3 in Arabidopsis. J Exp Bot. 2018;69:291122.

54. Kong L, Zhi P, Liu J, Li H, Zhang X, Xu J, et al. Epigenetic activation of Enoyl CoA reductase by an acetyltransferase complex triggers wheat wax biosynthesis. Plant Physiol. 2020;183:1250-67. 
55. Zhi P, Kong L, Liu J, Zhang X, Wang X, Li H, et al. Histone deacetylase TaHDT701 functions in TaHDA6-TaHOS15 complex to regulate wheat defense responses to Blumeria graminis f.sp. tritici. Int J Mol Sci. 2020;21:2640.

56. Larkin MA, Blackshields G, Brown NP, Chenna R, McGettigan PA, McWilliam H, et al. Clustal W and Clustal X version 2.0. Bioinformatics. 2007;23:294748.

57. Kumar S, Stecher G, Tamura K. MEGA7: Molecular evolutionary genetics analysis version 7.0 for bigger datasets. Molec Biol Evol. 2016;33:1870-74. 58. Letunic I, Bork Peer. Interactive tree of life (iTOL) v4: recent updated and new developments. Nucleic Acids Res. 2019;47: 256-259.

59. Bailey TL, Williams N, Misleh C, Li WW. MEME: discovering and analyzing DNA and protein sequence motifs. Nucleic Acids Res. 2006;34:369-73. 60. Deng WK, Wang YB, Liu ZX, Cheng H, Xue Y. Heml: a toolkit for illustrating heatmaps. Plos One. 2014;9:e111988.

\section{Tables}

Table 1. Basic physicochemical properties, Secondary structure prediction, and subcellular localization HATs Setaria italica

\begin{tabular}{|c|c|c|c|c|c|c|c|c|c|c|}
\hline & $\begin{array}{l}\text { Gene } \\
\text { name }\end{array}$ & Gene ID & Chromosome & Start & End & $\begin{array}{l}\text { Number } \\
\text { of } \\
\text { amino } \\
\text { acids }\end{array}$ & $\begin{array}{l}\text { Isoelectric } \\
\text { point }\end{array}$ & $\begin{array}{l}\text { Molecular } \\
\text { weight }\end{array}$ & $\begin{array}{l}\text { Instability } \\
\text { index }\end{array}$ & $\begin{array}{l}\text { Aliphatic } \\
\text { index }\end{array}$ \\
\hline SiHAG1 & SiHAT1 & Seita.1G016200.1 & 1 & 1408810 & 1415898 & 676 & 9.49 & 73946.55 & 64.49 & 64.91 \\
\hline SiHAC1 & SiHAT2 & Seita.1G093400.1 & 1 & 8226084 & 8238005 & 1722 & 8.41 & 193778.81 & 51.08 & 64.55 \\
\hline SiHAG2 & SiHAT3 & Seita.1G222200.1 & 1 & 30066271 & 30071465 & 707 & 5.1 & 77673.96 & 59.46 & 72.05 \\
\hline SiHAC2 & SiHAT4 & Seita.2G043800.1 & 2 & 3521084 & 3529967 & 1274 & 7.2 & 144323.76 & 53.25 & 67.2 \\
\hline SiHAC3 & SiHAT5 & Seita.2G154800.1 & 2 & 22217294 & 22239544 & 5068 & 5.85 & 563547.45 & 45.7 & 89.72 \\
\hline SiHAG3 & SiHAT6 & Seita.2G183200.1 & 2 & 27835799 & 27841209 & 470 & 4.99 & 52458.39 & 48.41 & 90.19 \\
\hline SiHAF1 & SiHAT7 & Seita.2G272400.1 & 2 & 37214286 & 37221484 & 1198 & 6.05 & 133781.16 & 57.59 & 77.19 \\
\hline SiHAG4 & SiHAT8 & Seita.2G299000.1 & 2 & 39265652 & 39271852 & 581 & 8.53 & 64096.4 & 71.38 & 55.52 \\
\hline SiHAG5 & SiHAT9 & Seita.2G326000.1 & 2 & 41360971 & 41372516 & 425 & 7.07 & 47425.44 & 36.14 & 84.49 \\
\hline SiHAG6 & SiHAT10 & Seita.2G328800.1 & 2 & 41595648 & 41603366 & 1254 & 9.41 & 137904.59 & 70.22 & 57.19 \\
\hline SiHAM1 & SiHAT11 & Seita.2G398400.1 & 2 & 46215552 & 46221344 & 441 & 7.21 & 50338.37 & 41.05 & 75.85 \\
\hline SiHAF2 & SiHAT12 & Seita.3G366500.1 & 3 & 46981012 & 46995352 & 1715 & 5.25 & 193378.51 & 51.49 & 75.12 \\
\hline SiHAG7 & SiHAT13 & Seita.4G027300.1 & 4 & 1781574 & 1785951 & 346 & 6.65 & 39271.65 & 45.18 & 73.29 \\
\hline SiHAF3 & SiHAT14 & Seita.4G200100.1 & 4 & 31872825 & 31887569 & 1763 & 5.31 & 198370.34 & 51.62 & 72.04 \\
\hline SiHAC4 & SiHAT15 & Seita.4G271400.1 & 4 & 38804311 & 38813976 & 1569 & 8.68 & 177479.4 & 51.19 & 69.97 \\
\hline SiHAG8 & SiHAT16 & Seita.5G245000.1 & 5 & 30720688 & 30724992 & 353 & 9.2 & 38076.51 & 64.54 & 58.73 \\
\hline SiHAC5 & SiHAT17 & Seita.5G411200.1 & 5 & 43527986 & 43531785 & 427 & 8.69 & 47760.46 & 50.43 & 89.95 \\
\hline SiHAC6 & SiHAT18 & Seita.5G418300.1 & 5 & 44110004 & 44117354 & 1161 & 6.67 & 131122.61 & 50.77 & 69.53 \\
\hline SiHAG9 & SiHAT19 & Seita.6G009400.1 & 6 & 621314 & $628373 \mathrm{f}$ & 612 & 5.33 & 66820.85 & 55.08 & 51.88 \\
\hline SiHAG10 & SiHAT20 & Seita.6G078100.1 & 6 & 7010285 & 7016455 & 775 & 4.96 & 85422.61 & 58.04 & 71.99 \\
\hline SiHAG11 & SiHAT21 & Seita.7G143400.1 & 7 & 23254046 & 23258366 & 572 & 8.82 & 63909.5 & 38.24 & 85.79 \\
\hline SiHAG12 & SiHAT22 & Seita.8G053600.1 & 8 & 4674484 & 4682125 & 583 & 5.65 & 64372.42 & 50.96 & 70.74 \\
\hline SiHAG13 & SiHAT23 & Seita.9G263000.1 & 9 & 21742690 & 21747886 & 515 & 6.25 & 56898.58 & 45.59 & 63.38 \\
\hline SiHAG14 & SiHAT24 & Seita.9G553300.1 & 9 & 57060719 & 57066495 & 714 & 9.86 & 77219.48 & 59.54 & 56.36 \\
\hline
\end{tabular}

\section{Figures}

\section{Figure 1}

Chromosome locations of histone acetylation genes (HATs) in Setaria italica. Chromosomal location was performed on 24 histone acetylation gene family members in S. italica. 

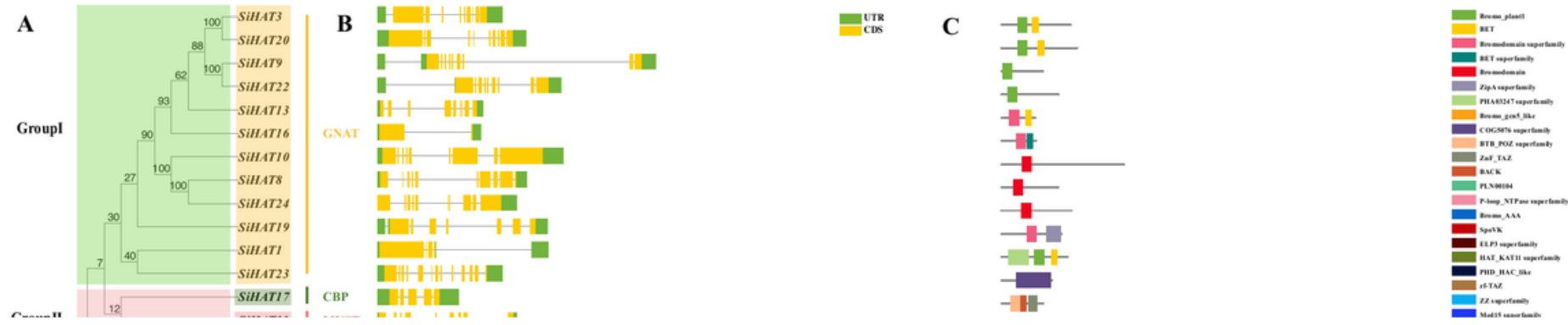

Figure 2

Histone acetylation gene (HAT) structure and conserved domains in Setaria italica

Figure 3

Phylogenetic analysis and Collinearity analysis of histone acetylation proteins (HATs) in Arabidopsis thaliana, Oryza sativa, and Setaria italica.

A

GroupI

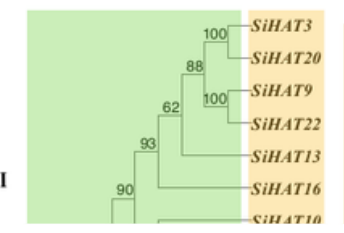

B

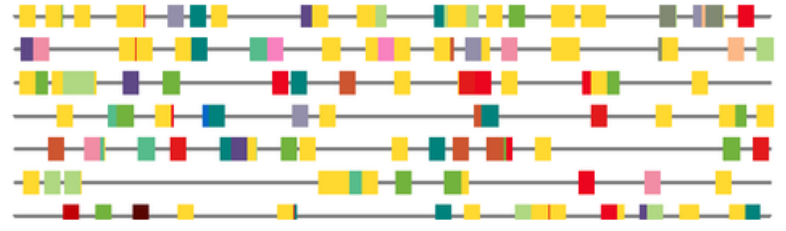

the MeJA-responsive element

zein metabolism regulative element

light responsive element

the anaerobic inductive element

gibberellin-responsive element

MYB binding site involved in drought-inducibility

anoxic specific inducibility element

the abscisic acid responsive element

Figure 4

Prediction of cis-acting elements in promoter of histone acetylation genes (HATs) in Setaria italica

Figure 5

Relative expression patterns of histone acetylation genes (HATs) in different tissues of Setaria italica. Heat maps reflect the fragments per kilobase of transcript per million mapped fragments (FPKM) of HATs. Color from red to blue indicates high to low expression. 
Figure 6

Expression analysis of histone acetylation genes (HATs) in Setaria italica under low nitrogen stress. Low nitrate stress time represented by 10 min, 30 min, 2,8 , 24 and 7 h, NN and LN represent normal and low nitrogen treatment, respectively. Leaf and Root describe tissues sampled.

Figure 7

Expression analysis of histone acetylation genes (HATs) in Setaria italica under low phosphorus stress. Low phosphorus stress time represented by $0.5,2,6$, 12, 24, and $72 \mathrm{~h}$. NP and LP represent normal and low phosphorus treatments, respectively. Leaf and Root describe tissues sampled.

Figure 8

Expression analysis of HATs genes in foxtail millet under drought stress. Control and Drought represented Control and Drought treatment groups, respectively. $\mathrm{R}$ (AN04) and S (Yugu1) represent drought resistant and drought sensitive varieties, respectively. Light and Dark represent different sampling time and illumination: (light) illumination, (dark) no illumination.

Figure 9

Expression analysis of HATs genes in foxtail millet under salt-alkali stress. CK and SAS represent control group and salt-alkali treatment group, respectively. $\mathrm{R}$ (B103) and S (B355) represent salt-resistance and salt-sensitive varieties, respectively. T1 and T2 represent different sampling tissues: T1 is Seedlings germinating for 3 days and $\mathrm{T} 2$ is one-tip-two-leaf Seedlings.

\section{Figure 10}

Expression analysis histone acetylation genes (HATs) in Setaria italica under Sclerospora graminicola infection stress. CK and T represent the control and infection treatment groups, respectively. R and $\mathrm{S}$ represent resistant and sensitive varieties, respectively. Numbers 3,5 , and 7 represent leaf stages at different sampling times.

\section{Supplementary Files}

This is a list of supplementary files associated with this preprint. Click to download.

- SupplementalTable1.InformationofhistoneacetylationgeneHATstructureandconserveddomaininSetariaitalica.docx

- SupplementalTable2.OnetooneorthologousrelationshipsbetweenSetariaitalicaandothertwoplantspecies..docx

- SupplementalTable3.PredictionofcisactingelementsinpromotersofhistoneacetylationgenesHATsinSetariaitalica.docx

- SupplementalTable4.Detailsof23tissuessampledinthespatialandtemporalexpressionexperiment.docx 